\title{
Inter- and intra-fractional stability of rectal gas in pelvic cancer patients during MRIgRT
}

DOI:

10.1002/mp.14586

Document Version

Accepted author manuscript

Link to publication record in Manchester Research Explorer

\section{Citation for published version (APA):}

Shortall, J., Vasquez Osorio, E., Mackay, R., Song, Y. P., Kirkby, K., Cree, A., Dubec, M., Chuter, R., Mcwilliam, A., Price, G., \& Van Herk, M. (2020). Inter- and intra-fractional stability of rectal gas in pelvic cancer patients during MRIgRT. Medical Physics. https://doi.org/10.1002/mp.14586

\section{Published in:}

Medical Physics

\section{Citing this paper}

Please note that where the full-text provided on Manchester Research Explorer is the Author Accepted Manuscript or Proof version this may differ from the final Published version. If citing, it is advised that you check and use the publisher's definitive version.

\section{General rights}

Copyright and moral rights for the publications made accessible in the Research Explorer are retained by the authors and/or other copyright owners and it is a condition of accessing publications that users recognise and abide by the legal requirements associated with these rights.

\section{Takedown policy}

If you believe that this document breaches copyright please refer to the University of Manchester's Takedown Procedures [http://man.ac.uk/04Y6Bo] or contact uml.scholarlycommunications@manchester.ac.uk providing relevant details, so we can investigate your claim.

\section{OPEN ACCESS}




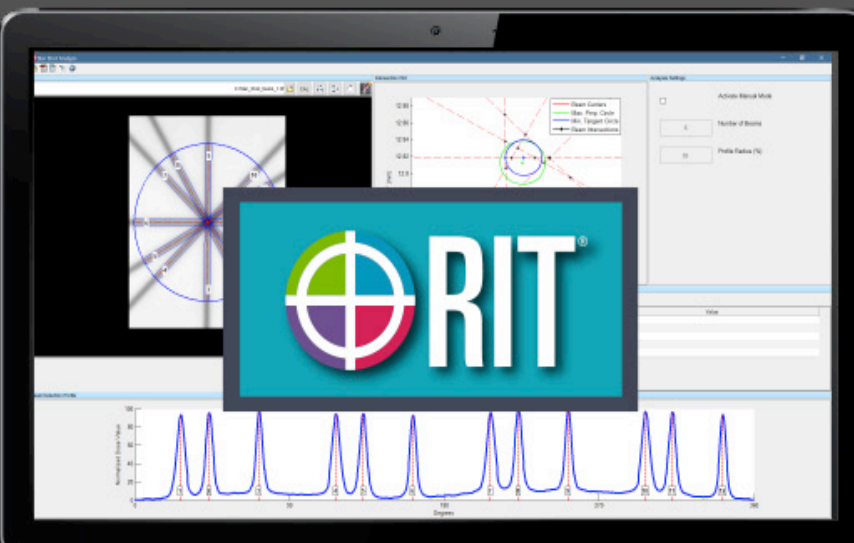

SEE WHAT'S NEW IN MEDICAL PHYSICS' LEADING QA SOFTWARE THE RIT FAMLLY OF PRODUCTS VERAONON 6.9

UTILIZE RIT'S LATEST \& GREATEST UPGRADE FOR PRECISION QA NEW FEATURE HIGHLIGHTS INCLUDE:

- 60-Leaf MLC Support for Varian RapidArc ${ }^{\circledast}$ Tests
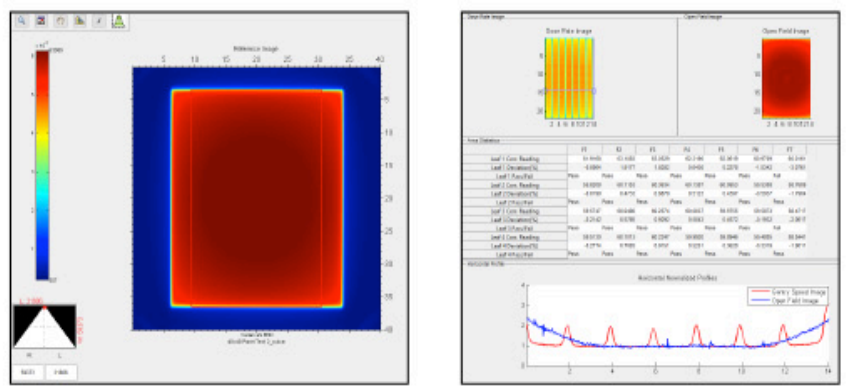

Utilize the 60-Leaf MLC analysis for Varian RapidArc ${ }^{\circledast}$ tests, in addition to the traditional 40-leaf MLC, which allows for expanded support of a wider range of Varian LINACs. The SID is now automatically extracted from DICOM images.

- Convenient, Cloud-Based Software Licensing Utilize floating license capability, local to your facility's network with the Local License Server license configuration option.

- Couch Placement Phantom Analysis Perform couch placement analysis for PTW EPID and Primus ${ }^{\oplus}$-L phantoms, providing an analysis that is closer to realistic patient positioning.

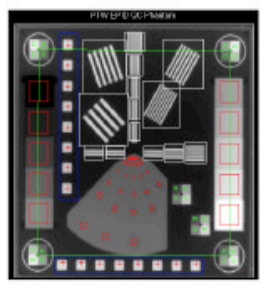

- Enhanced CATPHAN ${ }^{\circledR}$ Low-Contrast Module Features In the CATPHAN ${ }^{\circledR}$ Low Contrast module, select among Contrast-to-Noise analysis options for the phantom.

Easily select the "CNR Only" analysis type to analyze the CNR between the reference region and the local background region of a CATPHAN ${ }^{\circledR}$ Low Contrast image.
- Enhanced TomoTherapy ${ }^{\circledast}$ Registration Easily perform exact dose comparisons for TomoTherapy patient QA. The test uses a TomoTherapy ${ }^{\circledast}$ plan, a dose map, and a film to determine position and dose accuracy, now with expanded support for additional Accuray treatment planning systems.

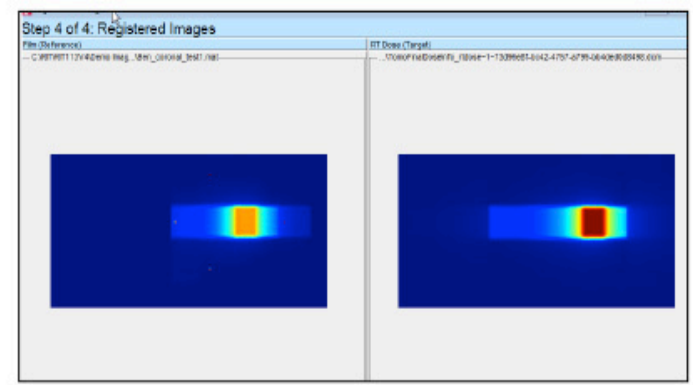

- Options for Las Vegas EPID Phantom QA Guidelines Enjoy increased flexibility on acceptance criteria (TG-58 or Varian QA guidelines) when analyzing images from the Las Vegas EPID phantom. The QA guideline determines which of two tests will be performed in the analysis: (1) Contrast and Spatial Resolution and/or (2) Image Quality.

- Diagnostic TOR-18 Phantom Analysis Perform diagnostic TOR-18 phantom analysis on images captured with high-resolution fluoroscopy machines.

\section{- DICOM Anonymizer}

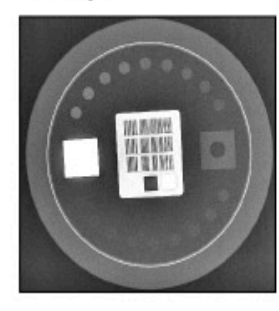
Use the new DICOM Anonymizer feature to automatically remove sensitive and confidential medical information from any DICOM file.

\section{REQUEST A PERSONAL DEMO TODAY}


DR. HAO FENG (Orcid ID : 0000-0003-1038-1495)

PROF. LILI HUANG (Orcid ID : 0000-0002-7085-7646)

Article type : Research Article (MP)

Full title: Inter- and intra-fractional stability of rectal gas in pelvic cancer patients during MRIgRT

\section{Running title: Stability of rectal gas during MRIgRT}


McWilliam $^{1,2}$, K Kirkby $^{1,2}, \mathrm{R}$ Mackay ${ }^{1,2}, \mathrm{M}$ van Herk ${ }^{1,2}$

1. The University of Manchester Department of Cancer Sciences, Manchester UK

2. The Christie NHS Foundation Trust, Manchester UK

Corresponding author contact: Eliana Vasquez Osorio

Email: eliana.vasquezosorio@manchester.ac.uk

Postal address: Dept. 58, The Christie NHS foundation Trust,

Wilmslow Road,

Manchester

M20 4BX

Author contacts:

jane.shortall@postgrad.manchester.ac.uk

Anthea.cree@christie.nhs.uk

yeepei.song@christie.nhs.uk

Michael.dubec@christie.nhs.uk

Robert.chuter@christie.nhs.uk

Gareth.price@manchester.ac.uk

This article has been accepted for publication and undergone full peer review but has not been through the copyediting, typesetting, pagination and proofreading process, which may lead to differences between this version and the Version of Record. Please cite this article as doi: 10.1002/MP.14586

This article is protected by copyright. All rights reserved 
Alan.mcwilliam@manchester.ac.uk

Karen.kirkby@manchester.ac.uk

Ranald.mackay@christie.nhs.uk

Marcel.vanherk@manchester.ac.uk

Keywords: Cancer, Radiotherapy, Image guided Radiotherapy, IGRT, MR-guided Radiotherapy, MRgRT, organ movement

Purpose: Due to the Electron Return Effect (ERE) during Magnetic Resonance Imaging guided Radiotherapy (MRIgRT), rectal gas during pelvic treatments can result in hot spots of overdosage in the rectal wall. Determining the clinical impact of this effect on rectal toxicity requires estimation of the amount and mobility (and stability) of rectal gas during treatment. We therefore investigated the amount of rectal gas and local inter- and intra-fractional changes of rectal gas in pelvic cancer patients.

Methods: To estimate the volume of gas present at treatment planning, the rectal gas contents in the planning CT scans of 124 bladder, 70 cervical and 2,180 prostate cancer patients was calculated.

To estimate inter- and intra-fractional variations in rectal gas, 174 and 131 T2-w MRIs for six cervical and eleven bladder cancer patients were used. These scans were acquired during four scan-sessions ( 20-25 minutes each) at various time-points. Additionally, 258 T2-w MRIs of the first five prostate cancer patients treated using MRIgRT at our centre, acquired during each fraction, were analysed. Rectums were delineated on all scans.

The area of gas within the rectum delineations was identified on each MRI slice using thresholding techniques. The area of gas on each slice of the rectum was used to calculate the inter- and intra-fractional group mean, systematic and random variations along the length of the rectum.

The cumulative dose perturbation as a result of the gas was estimated. Two approaches were explored: accounting or not accounting for the gas at the start of the scan-session.

Results: Intra-fractional variations in rectal gas are small compared to the absolute volume of rectal gas detected for all patient groups. I.e., rectal gas is likely to remain stable for periods of 20-25 minutes. Larger volumes of gas and larger variations in gas volume were observed in bladder cancer patients compared with cervical and prostate cancer patients. 
For all patients, local cumulative dose perturbations per beam over an entire treatment in the order of $60 \%$ were estimated when gas had not been accounted for in the daily adaption. The calculated dose perturbation over the whole treatment was dramatically reduced in all patients when accounting for the gas in the daily set-up image.

Conclusion: Rectal gas in pelvic cancer patients is likely to remain stable over the course of an MRIgRT fraction, and also likely to reappear in the same location in multiple fractions, and can therefore result in clinically relevant over-dosage in the rectal wall. The over-dosage is reduced when accounting for gas in the daily adaption.

\section{Introduction}

With superior soft tissue contrast to Cone-Beam Computed Tomography (CBCT) and the potential for real-time imaging, Magnetic Resonance Imaging guided Radiotherapy (MRIgRT) shows promise for pelvic sites including cervix, bladder and prostate [1]-[6]. The potential for real-time Adaptive Radiotherapy (ART) could help us to achieve more accurate treatment delivery for pelvic sites, which are subject to large and irreproducible inter- and intra-fractional anatomical variations [5]-[7].

Variations in rectal gas have been shown to influence target coverage during conventional cervical, bladder and prostate cancer radiotherapy [8]-[13]. Furthermore, due to the Electron Return Effect (ERE), there is a potential for dose perturbation in the rectal wall during MRIgRT treatment of these sites [14]. The ERE occurs when dose depositing electrons, under the influence of Lorentz forces, cross a tissue-air boundary and return back into the tissue they came from. This results in sizable areas of increased dose on the beam entry side of the tissue boundary and, due to a reduced number of electrons travelling forwards, areas of reduced dose on an opposing tissue boundary [4], [15], [16]. It is estimated that large volumes of rectal gas in the path of a single treatment beam can increase Dmax in the rectal wall by up to $60 \%$, potentially increasing the risk of grade $2+$ rectal toxicity [14].

The total dosimetric effect of rectal gas during MRIgRT depends on the beam configuration and the proportion of the treatment for which the gas is stable in a particular place. The longer gas is stable for during a fraction (intra-fraction), and the more it appears in the same place in multiple fractions (inter-fraction), the larger the total dosimetric effect. Also, in the case of hypofractionation, the total dose effect is more influenced by gas appearing in one of the fractions [14], [17]-[19]. 
Further, although daily adaption is performed for MRIgRT using a daily MRI image taken at the beginning of each fraction, several workflows re-optimise the daily dose using the average electron density from delineated structures in the patients' planning Computed Tomography (CT) scan [20], [21]. This means that inhomogeneity's within structures, such as rectal gas, and interfractional variations in rectal gas are neglected in the daily plan [22]-[24]. It also means that the electron density in the rectal structure will systematically be assumed to be lower if large amounts of gas were present in the planning CT, and higher if little or no gas was present.

Given the potential dose implications in the rectum due to gas during MRIgRT, it is important to assess the likelihood that large volumes of rectal gas remain stable over a treatment fraction or course to result in clinically concerning over-dosage in the rectal wall or under-dosage of the target.

Although some work has been done to assess rectal movement and its influence on target coverage [8]-[12], [25], little work has been done to assess the variations of rectal gas on inter- or intra-fractional timescales, and it is widely accepted to be unpredictable and irreproducible [8], [26]. This work uses large numbers planning CT scans to assess gas volumes, and repeat MRI scans to assess the local inter- and intra-fractional variability of rectal gas in patients with cervical, bladder and prostate cancer. We further explore the dosimetric effect of accounting or not accounting for rectal gas in the daily adaption.

\section{Method}

\section{Patient Images}

First, 2,374 planning CT scans of cervical, bladder and prostate patients were used to assess the volume of rectal gas on a population level (ethical approval ref. 17/NW/0060). To study inter- and intra-fractional rectal gas variation, 563 repeat T2-w MRI scans of 6 cervical (ethical approval ref. 17/NW/0300), 11 bladder (ethical approval ref. 18/NW/0352) and 5 prostate cancer patients were analysed. The prostate MRI images were acquired during the treatment fractions of the first five patients treated using MRIgRT at our centre. For consistency, we will refer to all MRI studies and MRI-Linac fractions as scan-sessions in this paper. The details of the scans used are summarised in Table 1.

This article is protected by copyright. All rights reserved 
Analysis

Calculating the Volume of Rectal Gas

First, the total volume of gas within the rectum delineations in the large cohort of planning CT scans was calculated using thresholding techniques, i.e., all CT voxels with pixel value less than $500 \mathrm{HU}$ were considered as gas.

Then the rectum was outlined according to clinical standard from the sigmoid colon to the anal canal on each MRI scan using a research version of the RayStation Treatment Planning System (TPS) (version 6.99, RaySearch Laboratories, Sweden). A radiation oncologist either drew or reviewed the outlines.

Slices where rectal delineations were present across all time-points in a scan-session were selected for analysis. The length of the rectum in the selected slices was normalised between 0 (most cranial) and 1 (most caudal) across all scans for all patients (Figure $1 \mathrm{~A}$ ). It was assumed that the normalised slices corresponded to the same anatomical level on the rectum in each timepoint, and that these levels were also comparable between patients, allowing a population level analysis. The length of rectums analysed and the length of rectums excluded from analysis due to our normalisation method, i.e. due to variation in rectum length during each scan-session, were also calculated and reported.

The intra-scan-session variability in rectal length was defined as the mean of the standard deviation (sd) of the rectal length over a scan-session. Inter-scan-session variability was defined as the mean of the sd of the mean rectal length per scan-session. The inter-patient variability was defined as the sd of the average rectal length per patient over all scan-sessions.

For each time-point, $X\{X=1, \ldots, N\}$, in each scan-session, the area of gas within each slice of the rectal delineations, $A_{X}$, was determined using thresholding (Figure $1 \mathrm{~B}$ ). The threshold was set to 60 , and was determined based on the pixel intensity of gas in the MRI scans. $A_{x}$ for each scansession was plotted against normalised rectum length to show the profile of rectal gas along the rectum (Figure $1 \mathrm{C}$ ).

Quantifying Inter- and Intra-fractional Rectal Gas Variations

Patients were grouped by disease site. To calculate the local inter- and intra-fractional variation of gas for each patient group, we adapted the statistical methodology for margin calculation developed by van Herk et al. [27]. The group mean, $M$, systematic, $\Sigma$, and random, $\sigma$, and intra- 
fractional random, $\sigma_{f}$, variation on each normalised slice of the rectum was calculated for interand intra-fractional changes using standard the methods presented in Figure $1 \mathrm{~S}$ in the supplementary materials.

$M$ is defined as the mean of the mean, and shows the average amount of gas across the patient group, $\Sigma$ is the standard deviation of the mean, and shows the spread of the average amount of gas per patient across the patients and $\sigma$ and $\sigma_{f}$ are root-mean-square of the standard deviations, and indicate the average inter-fractional variation across scan-sessions or intra-fractional variation across time-points respectively.

To evaluate inter-fractional variations, $A_{\text {inter, }}$, we used the mean area of gas in each slice over all time-points per scan-session (Figure $1 \mathrm{~S}$ supplementary material):

$$
A_{\text {inter }}=\left(\bar{A}_{x}\{X=1, \ldots, N\}\right)
$$

For intra-fractional variations, $A_{\text {intra, }}$, the change in gas area between time-point 1 , i.e. the daily set-up image used for plan adaption during MRIgRT, and the rest of the time-points, $\{X=2 \ldots N\}$ was used (Figure 1S supplementary material):

$$
A_{\text {intra }}=\left(A_{X}\{X=2, \ldots, N\}-A_{1}\right),
$$

The intra-fractional random variation, $\sigma_{f}$, was calculated using $\sigma$ for each patient and then averaging over all patients (Figure 1S supplementary material).

Note that these calculations were performed on each image slice included in the analysis, and the full set of statistics was calculated separately for each patient group. To account for the uncertainty in the rectal lengths, a Gaussian filter with a width corresponding to the combined inter- and intra-scan-session variability was applied to the statistical plots [28].

Intra-fractional Dose Effects due to Rectal Gas

Analytically estimating dose perturbation

The dosimetrical effect of accounting or not accounting for and accounting for rectal gas in the daily plan adaption was explored. The local dose perturbation, $\Delta D_{\%(X)}$, caused by the gas detected in each scan-session was calculated first assuming the gas was not considered in the daily adaption. $\Delta D_{\%(X)}$, was then re-calculated assuming that the dosimetric effects of gas present at the beginning of each scan-session were mitigated in the daily adaption. 
The local dose perturbation, $\Delta D_{\%(X)}$, due to the gas detected in each time-point, $X\{X=1, \ldots, N\}$, was calculated using equation 1, an adapted version of an equation previously presented in [29]. The equation calculates the dose perturbation, $\Delta D_{\%}$, at angles $\theta, \Phi$ around spherical air cavities (up to $1 \mathrm{~cm}$ distance, $d$, from the cavity surface) for the Elekta Unity system with a $1.5 \mathrm{~T}$ transverse magnetic field and $7 \mathrm{MV}$ photon spectrum irradiated with a single beam. The equation was derived in [29] using data fitting techniques, and describes the dosimetrical effects of ERE and attenuation differences for a single beam centred on a spherical air cavity using a modulated sinusoidal and a Gaussian function for ERE and attenuation effects respectively. The amplitude of both effects depends on the cavity radius, $r$, either logarithmically (ERE) or linearly (attenuation differences). This equation is designed for and has been validated on spherical and cylindrical air cavities with $r$ between the range of $0.5 \mathrm{~cm}$ and $7.5 \mathrm{~cm}$.

We modified the logarithmic term in the original (heuristic) equation to allow it to be applied where gas cavities may be $<0.5 \mathrm{~cm}$. The dose perturbation around air cavities with a diameter smaller than the radius of curvature of secondary electrons under the influence of Lorentz forces $(<0.5$ $\mathrm{cm}$ diameter) is negligible [14], [18]. We therefore modified equation 1 to generate zero dose perturbation where the diameter of rectal gas tended towards zero. We validated our equation on spherical air cavities $(0.5 \mathrm{~cm}<$ diameter $<7.5 \mathrm{~cm})$ by calculating the predicting error; the difference between the dose perturbation predicted by our equation and that calculated using Monte Carlo dose calculations ( $\Delta \mathrm{D} \%$ Predict - $\Delta \mathrm{D} \%$ MonteCarlo). Table $1 \mathrm{~S}$ in the Supplementary material summarises the predicting error for various diameters of air cavity between 0.5 and 7.5 $\mathrm{cm}$. The mean and standard deviation of the predicting error across all air cavities was $\leq+/-0.9 \%$ and $\leq+/-7.7 \%$ respectively.

\section{Equation 1}

$$
\begin{gathered}
\Delta D_{\%}(\theta, \Phi, r, d)=(-55 \ln (r+1)) * e^{-3.71 d} * \sin (1.04 \theta-1.97) * \sin (1.05 \Phi+6.20)+7.14 r(\exp \\
{\left[-\left(\frac{(\theta-3.02)^{2}}{2 * 0.37^{2}}\right)-\left(\frac{(\Phi-1.59)^{2}}{2 * 0.79^{2}}\right)\right]-1.29 .}
\end{gathered}
$$

The local relative dose perturbation, $\Delta D_{\%(X)}$, assuming the original dose to be 1 , on the internal surface of the rectal wall (that is $d=0 \mathrm{~cm}$ in the equation) was calculated.

The equation was applied to each normalised slice, assuming each slice to be the central plane of a sphere, creating a dose map of the internal surface of the rectal wall. The gas on each slice was assumed to be circular, with its whole circumference directly touching the internal surface of the rectal wall.

This article is protected by copyright. All rights reserved 
There are a very large number of permutations of beam configurations, rectal anatomy and gas location that cannot possibly be explored here. We therefore estimated the dosimetric consequences for the "worst-case" scenario, assuming that rectal gas lies in the path of a single beam of the treatment plan for the whole treatment course. This assumption was deemed reasonable as we compare like-for-like to show the impact of accounting for gas in the daily adaption. Further, we consider the rectum as an OAR in this work, and so multiple beams are not expected to be focussed on the rectum.

Implementing the analytical equation

To determine the cumulative local dose perturbation over a scan-session, $\Delta D_{\%(\text { Cumulative)Scan-session, }}$ the local $\Delta D_{\%(X)}$ at each "beam-on" time-point was calculated and associated with a time period, $f(t)$. To simulate the "beam on" time during a typical treatment fraction, only the last 6-7 minutes of each scan-session were used.

The associated time period, $f(t)$, was defined as a fraction of the total time of the "beam on" time and assumed to spread halfway between the neighbouring two time-points, TP:

Equation 2

$$
f(t)=\frac{(t(T P i+1)-t(T P i))+(t(T P i)-t(T P i-1))}{2\left(t\left(T P_{N}\right)-t\left(T P_{\text {Beamon }}\right)\right)} .
$$

The calculated local $\Delta D_{\%(X)}$ for each time-point was then multiplied with the associated $f(t)$ to

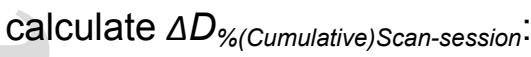

Equation 3

$$
\Delta D_{\%(\text { Cumulative })_{\text {Scan-session }}}=\sum_{T P_{\text {Beam } O n}}^{T P_{N}} f(t) \Delta D_{\%(X)}
$$

For those scan-sessions with only one time-point associated with them, the gas was assumed to remain stable for the entirety of the "beam on" time. For scan-sessions with two time-points, it was assumed that the gas per time-point was stable for half of the "beam on" time.

Note that here we assume that the daily workflow uses average electron density over-rides, and so any gas present at the beginning of the scan-session (time-point 1 ) is neglected [23].

To determine the effect of rectal gas when re-optimising the dose for each MRIgRT fraction, the above process was repeated but this time using the dose estimated with gas present at the 
beginning of each scan-session (time-point 1) as a baseline, and the relative change in dose due to changing gas during each scan-session used to calculate $\Delta D_{\%}$ :

$$
\begin{aligned}
\text { Equation } 4 \\
\Delta D_{\%(\text { Cumulative })_{\text {Scan - session_adapt }}}=\sum_{X_{\min }}^{X_{N}} f(t) \Delta D_{\%}\left(A_{X}-A_{1}\right)
\end{aligned}
$$

where

$$
\{X=2, \ldots, N\}
$$

Note that it is the dose effects from each time-point that are combined rather than the volume of gas in each time-point. Scan-sessions with only one time-point were excluded from this stage of the analysis.

The cumulative $\Delta D_{\%(C u m u l a t i v e) \text { Total }}$ and $\Delta D_{\% \text { (Cumulative)Total_adapt }}$ over all scan-sessions for each patient was calculated using:

Equation 5

$$
\Delta D_{\%(\text { Cumulative }) \text { Total }}=\sum_{\text {Scan }- \text { Session }_{1}}^{\text {Scan }- \text { session }_{N}} f(t) \Delta D_{\%(\text { Cumulative }) \text { Scan }- \text { session }}
$$

where the time period, $f(t)$, is now

$$
f(t)=1 / N
$$

where $N$ is the total number of scan-sessions per patient. This is equivalent to simulating a treatment with 4 fractions for the cervix and bladder cases, or a 20 fraction treatment for the prostate cases.

\section{Results}

Rectal Gas Volume: Planning CT vs MRI

Figure 2 shows the distribution of the total volume of gas detected in the planning CT scans of the 2,374 patients (70 cervical, 124 bladder and 2,180 prostate cancer). $97.8 \%$ of the patients had $<50 \mathrm{~cm}^{3}$ of rectal gas in their planning CTs, and almost half of the patients $(43 \%)$ had $<1 \mathrm{~cm}^{3}$. 
The largest volumes of gas were seen in the bladder patients. The bladder patients had a mean rectal gas volume of $9.8 \mathrm{~cm}^{3}$, and four of the patients $(3 \%)$ had $>50 \mathrm{~cm}^{3}$ of rectal gas in their planning CTs (maximum=129 $\mathrm{cm}^{3}$ ). The prostate patients had the least gas with mean volume of $7.0 \mathrm{~cm}^{3}$ (44 patients $\left(2 \%\right.$ ) had $>50 \mathrm{~cm}^{3}$, maximum $=106 \mathrm{~cm}^{3}$ ). The cervix patients lay between the bladder and prostate groups with a mean gas volume of $7.6 \mathrm{~cm}^{3}\left(\max =41.3 \mathrm{~cm}^{3}\right)$. The difference between the distribution of rectal gas volume between three groups is border-line statistically significant $(p=0.05$ (Kruskal-Wallis test $))$.

Figure 3 shows the area of gas along the length of the rectum, which has been normalised from 0 (most cranial) to 1 (most caudal), for cervical cancer patient 1 . No rectal gas was detected in this patients' planning CT.

Clear inter-fractional variations are seen across each scan-session for this patient. Further, although the total volume of gas remains quite stable over scan-session 1 , the gas moves locally intra-fractionally towards the end of the scan-session, which happens to be the "beam on" period (time-points 5-7). For scan-sessions 3 and 4 there is a smaller and more stable volume of gas.

Similar plots for all other patients are presented in supplementary material (Appendix A-C supplementary material).

\section{Rectum Length Variation}

Table 2 summarises the results related to rectum length variation. All variations are relatively small; inter- and intra-scan-session variations were $\leq 0.71 \mathrm{~cm}$, and inter-patient variations are $\leq 1.46 \mathrm{~cm}$, which corresponds to $\sim 10 \%$ of the mean average length. These results indicate that the variability of the rectal length and the sensitivity of the methods used for analysis in this work is small.

We have applied a Gaussian filter with a width of 0.1 to account for the rectum variability uncertainty. This value was derived from the inter- and intra-scan-session variability. Note that, as the inter-patient results are a form of descriptive statistics, where the length is normalised between 0 and 1 to indicate the beginning and end of the rectum, the absolute length of the rectum has no impact on the results and was therefore not included in the Gaussian blurring. Inter- and Intra-fractional Variations in Rectal Gas during MRI Scan-Sessions

Figure 4 shows the local statistics $\left(M, \Sigma, \sigma\right.$ and $\left.\sigma_{f}\right)$ for inter- (panels A-C) and intra-fractional (panels D-F) variations in rectal gas, calculated for each patient group. A Gaussian filter with a width $=0.1$ (10\% of the normalised rectal length) was applied to account for variability in the rectal length during the analysis. As would be expected, inter-fractional variations are larger than 
intra-fractional variations. For all patient groups, the intra-fractional changes are small compared with the absolute area of gas in each slice (Figure 3 and Appendix A-C supplementary material). This indicates that rectal gas in all patients is relatively stable.

Differences are observed between patient groups. Both inter- (up to $8.5 \mathrm{~cm}^{2} / \mathrm{slice}$ ) and intrafractional (up to $5 \mathrm{~cm}^{2} / \mathrm{slice}$ ) variations are largest in bladder cancer patient patients, and are around five times larger than those seen in the cervical and prostate cancer patients, who had similar inter- and intra- fractional variations $\left(\sim 1-2.5 \mathrm{~cm}^{2} /\right.$ slice $)$.

There are also differences between patient groups in the distribution of gas along the length of the rectum. For cervical and prostate cancer patients, both inter- and intra-fractional changes are larger towards the cranial aspect of the rectum, and gas appears more stable at the caudal end. The opposite is observed for the bladder cancer patients; inter-fractional variations are largest in the centre of the rectum length and intra-fractional variations are largest towards the caudal end. Of course, this part of the analysis is based on small patient numbers, so there may be an element of chance in these differences.

Dose Effects of Rectal Gas

\section{Single Scan-Session}

Figures 5 and 6 show the distribution of estimated local dose perturbation for a single beam on the internal surface of the rectum during four scan-sessions when gas is not (Figure 5) and is (Figure 6) accounted for in the daily adaption for cervical patient 1. For all scan-sessions, large areas of dose of around $\pm 70 \%$ (Figure 5 ) were reduced to almost zero when gas is accounted for in the daily plan. Equivalent plots for all patients, where similar observations are true, are presented in Appendix A - C in supplementary material.

The box plots in Figure 7 panels A-C show the cumulative dose perturbation on the internal surface of the rectal wall for each scan-session, $\Delta D_{\%(\text { Cumulative)Scan-session, for all patients when gas }}$ has not (red boxes) and has (blue boxes) been accounted for in the daily adaption. Is it clear that for all patients, the dose perturbation in each scan-session is greatly reduced when gas is accounted for in the daily adaption.

Total Treatment

The box plots in Figure 7 panels D-F show the cumulative local dose perturbation for a single beam on the internal surface of the rectal wall over each patients' total treatment, $\Delta D_{\%(\text { Cumulative)Total. }}$ As expected, $\Delta D_{\%(\text { Cumulative)Total }}$ is smaller than $\Delta D_{\%(\text { Cumulative)Scan-session, }}$ indicating that gas moves between scan-sessions. However, $\Delta D_{\%(\text { Cumulative)Total }}$ still ranges between $\pm \sim 60 \%$, suggesting that 
considerable volumes of gas reappear in the same location along the length of the rectum over multiple scan-sessions to result in clinically concerning dose perturbation over the total treatment course.

For all patients, the maximum dose perturbation over a full treatment course is reduced to around

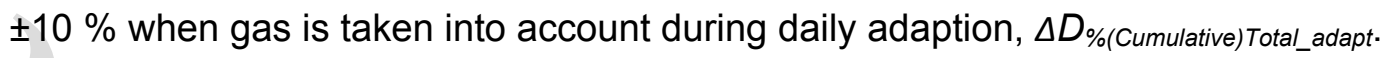

\section{Discussion}

This work aimed to quantify the local inter- and intra-fractional variation in rectal gas in pelvic cancer patients and estimate its dosimetric effect. We first assessed the likelihood that large volumes of rectal gas remain stable over the duration of an MRIgRT treatment thereby resulting in clinically relevant over-dosage due to ERE in the rectal wall. Then we explored the dosimetric effect of not accounting or accounting for the gas present in the daily set-up image. This study is conducted in light of work that indicates that rectal gas lying in the path of a treatment beam could increase the risk of rectal toxicity [14].

We adopt a similar methodology to map the rectum as Nijkamp et al., who quantify inter-fractional shape variation of the rectum for rectal cancer patients. However, we are the first to quantify interand intra-fractional variations of rectal gas, and included patients that have been treated with MRIgRT, in this kind of study [26], [27].

\section{Volume of Rectal Gas}

Patients generally had more rectal gas in the MRI scan-sessions than in their planning CT scans. This may be due to the longer imaging times involved with the MRI scan-sessions, or possibly due to the comfort of the imaging bed. Cervical cancer patients 1, 2 and 6 had no gas in their planning CT scans, yet all had gas in all four of their MRI scan-sessions (supplementary material Appendix A). Bladder cancer patients had the largest volumes of gas in both the planning CTs and MRIs.

Inter- and Intra-fractional Variations in Rectal Gas

For the MRI scan-sessions, local intra-fractional variations in rectal gas are small compared with the average volumes of gas detected $\left(2-6 \mathrm{~cm}^{2} / \mathrm{slice}\right)$. This suggests that gas present in the daily set-up MRI at the beginning of each 20-25 minute MRIgRT fraction is likely to remain stable throughout the treatment fraction. As a result, there is a significantly lower dose perturbation when re-planning based on the first image of each fraction. 
Alike Nijkamp et al., we observe differences in the total gas volume and gas variations between patient groups ( $p=0.05$, CT scan cohort). Whereas Nijkamp et al. report differences between male and females within the same disease site (rectal cancer), we group our patients by disease site. Nijkamp et al. find larger volumes and larger variations in gas for female rectal cancer patients compared with male rectal cancer patients [30], [31]. The only female patients in our study, the cervical cancer patients, had the least amount of rectal gas in both their planning CTs and MRI scan-sessions, compared with the bladder and prostate groups, who were all male. They also had smaller inter- and intra-fractional variations than the bladder cancer patients, but similar inter- and inter-fractional variations to the prostate group despite having around $50 \%$ less gas than the prostate patients (see Appendix A-C supplementary material).

The bladder cancer patients had the largest volumes of rectal gas in the planning CTs and MRI scan-sessions, and also the largest inter- and intra-fractional variations in rectal gas. This seems to be characteristic of bladder cancer patients [13], perhaps related to treatment protocols, the nature of the disease or the patient age and performance status, which is often worse for bladder patients.

An empty bladder protocol was used for all patients in this study. The cervix and bladder patients were instructed to drink $300-500 \mathrm{ml}$ of water immediately before each scan-session, and the bladder patients took Hyoscine Butylbromide (Buscopan) 30 minutes prior to each scan-session (Table 1). As the bladder and cervical patients had the most and least gas in the MRIs respectively, it is unlikely that bladder filling alone strongly influences rectal gas. However, the Buscopan that the bladder patients took could have influenced rectal gas behaviour.

The prostate patients, who had the mid volume of rectal gas during the MRIs, were not instructed to drink water or take Buscopan prior to their scan-sessions. In light of this, it could be that the bladder filling in the cervix patients limited rectal gas activity, and the Buscopan in the bladder patients increased it. It would be interesting to repeat this study to determine whether Buscopan influences rectal gas in cervical and prostate cancer patients in a similar was as in bladder cancer patients.

Further, it would be interesting to investigate if bladder filling combined with tumour location, i.e. if the tumour pushes against the rectum as the bladder fills, is influential on rectal gas. The differences between disease sites, particularly between the bladder and prostate patients, who were all male, suggest that characteristics such as tumour location may be more influential over the volume and stability of rectal gas than the patient's gender. This theory is supported by the same observations between the groups being observed in both the planning CT and MRI scan- 
sessions. It would be interesting to explore the influence of gender on rectal gas stability in bladder cancer patients by repeating this analysis with female patients. It would also be interesting to compare the rectal gas in female bladder patients to that in cervical cancer patients to investigate if tumour location is influential between these two disease sites.

Our results indicate that larger inter- and intra-fractional gas variations are observed at the cranial aspect of the rectum for cervical and prostate cancer patients (Figure $3 \mathrm{~A}$ ). This agrees with multiple other studies, that suggest that the caudal end of the rectum is more "anatomically fixed" [30]-[33]. However, our results also show that bladder patients have larger variations in the mid (inter-fractional) and more caudal (intra-fractional) aspect of the rectum. This disagrees with work reporting larger variations in bladder movement, due to bladder and rectal filling, in the cranial half of the bladder [13]. This could be due to the tumour location, gas passing out of the rectum during the scan-sessions, or a result of using a relatively small patient selection.

The average length of the rectums included in analysis was also different per patient group. The cervical patients had the longest rectums, and the bladder patients had the shortest with the largest variation (see Table 2). The variation in rectal length could be due to the rectum becoming shorter when expanding in the transverse direction due to filling.

The variation in the rectum length is small for all patient cohorts. Further, the largest variations in rectal gas (Figure 4), nor the regions of the rectum expected to receive a high dose, do not occur at the very extents of the analysed length of the rectum. Therefore, we believe the method used to normalise the rectums is not detrimental to results presented in this work.

It is worth noting that we assessed inter-fractional variation across all scan-sessions for all patients at once. Admittedly they do not appear to be grossly larger than intra-fractional changes in Figure $3 \mathrm{~A}-\mathrm{C}$. However, large changes occur between scan-sessions per patient when studying the gas profiles in Figure 3 and Appendix A-C in supplementary material. This, as well as changes between scan-sessions and the patient's planning $\mathrm{CT}$, should be incorporated into analysis in order to develop a model, similar to Hoogeman et al.'s., to predict if a patient is likely to be "gassy" and unstable during MRIgRT [34].

Dose Effects due to Rectal Gas

All patients benefited from accounting for gas present at the beginning of a scan-session during the daily adaption.

Some current MRIgRT workflows use the average electron density from each structure in the planning CT. Our results show that patients tend to have more gas during the MRI scan-sessions 
compared with their planning CTs. This suggests that the electron density for the rectal structure used to plan their whole treatment is likely to over-estimate the average electron density of the rectal structure in the patients' anatomy during treatment, which could result in over-dosage in the rectal wall.

Previous work by Chuter et al. assessing MRIgRT plan robustness for variations in sinus filing for head and neck cancer indicates that optimising using electron density over-rides to simulate an empty sinus cavity is preferable [35]. Our results could indicate that simulating large volumes of rectal gas when optimising a pelvic plan may be similarly beneficial. However, work adopting the same methodology as Chuter et al.'s would be needed to fully to explore this.

Alternatively, results we present in this paper show that if rectal gas is accounted for in the daily adaption process, dose perturbation due to intra-fractional variation in rectal gas are minimised. Incorporating the analytical equation we use in this work (equation 1) into the clinical workflow allows quickly alerting the treatment team if intra-fractional variations in rectal gas result in violation of dose constraints. This would help to optimise the on-line adaptive workflow.

It is appreciated that the analytical equation has only been validated using phantom data, and work should be done to further validate it using patient data. For clinical implementation, the equation should be paired with a tool to detect gas and calculate the local radius of curvature and the distance between gas boundaries, and be applied locally around the boundary of a gas cavity. In this work, areas of over-dosage are observed along the length of the rectum close to the entrance position of the beam $\left(0\right.$ and $\left.360^{\circ}\right)$, and under-dosage close to where the beam has left the rectum on the opposing side (Figures 5 and 6). This is because the beam angle is assumed to be the same for each scan-session. The location of the over-dosage could be moved for each treatment fraction, smearing out the cumulative dose perturbation over the whole treatment, by changing the beam angle of the beam travelling though the rectum. However, it is appreciated that this may not always be practical or achievable, particularly with the limited beam angles available for clinical treatment on the Elekta Unity system.

We consider gas in the path of a single beam in this paper, which is a "worst case" scenario. The total effect of gas in the path of multiple overlapping beams is a summation of the effect in each beam. Therefore, it is expected that the dose effects of rectal gas in the path of opposing beams would be lower than those we report in this paper [17].

It is appreciated that the rectum may not lie in the path of a single beam during pelvic treatments, and therefore dose perturbations we report may be overestimated. However, treatment beams are not expected to be focussed on the rectum when it is an OAR. As such, the likelihood that 
opposing beams totally cancel out the effects of rectal gas in a clinical treatment plan is questionable, as we see in [14] where the dose perturbation is reduced from $\sim 70 \%$ for a single beam to $\sim 20 \%$ for a 5-beam treatment.

There are a large number of permutations of beam configurations and air cavity locations, which cannot possibly be assessed here, and is beyond the scope of this paper. Work could be done to explore likely beam configurations covering the rectum. Clinical implementation of the heuristic equation we use to estimate dose effects should always involve applying it to each treatment beam and summing the effect, which can be done in real time [29].

The purpose of this work was to assess if rectal gas present at the beginning of a scan-session remains stable enough throughout a scan-session to result in clinically concerning dose perturbations in the rectum. Additionally, we looked at whether accounting for gas at the beginning of each scan-session is dosimetrically beneficial. As such, it is deemed that dosimetric estimation using a single beam to compare not accounting or accounting for gas is sufficient for the interests of this paper.

For interest however, to estimate the effect of rectal gas in the path of multiple beams, we propose to derive, a "geometry factor". Work by Shortall et al. showed that dose perturbations due to gas in the path of a five-beam plan are reduced to approximately one third of the dose perturbation in a single beam scenario, i.e. the "geometry factor" would be $\sim 0.3$ for a five-beam example plan [14]. Further, we observed a "geometry factor" of $\sim 0.1$ for a 15-beam plan using a web-based simulator incorporating MRIgRT uncertainties, produced by van Herk et al. [37]. With this in mind, the dose perturbations we report of $\sim 60 \%$ for a multiple fraction treatment would reduce to $\sim 18 \%$ for a five-beam scenario and $\sim 6 \%$ for a 15 -beam scenario. Further investigation deriving and validating this "geometry factor" for complete assessment of gas in the path of multiple beams is matter for future work.

The crucial issue is how much localised areas of over-dosage of up to $60-70 \%$ increases the risk of rectal toxicity. As discussed in [14], although it is thought that localised hotspots of 1cc receiving upwards of 70 Gy significantly increases the risk of grade 2+ late rectal toxicity [36][39], the question is by no means answered. The absolute dose perturbation due to rectal gas would of course depend on the proximity of the rectum to the tumour and other clinical planning parameters. In order to gain a greater understanding of the risk of increased rectal toxicity during MRIgRT, patient studies recording symptoms of acute side effects such as incontinence should be recorded, and, along with accurate dose accrual assessment, appropriate follow up should be implemented. 
The target coverage should also be kept in mind in light of large inter-fractional gas variations we report in this work. Variations in rectal filling has been shown to influence target coverage for bladder [13] and prostate cancer [40]. With this in mind, and the additional implication of rectal gas during MRIgRT, treating with an empty rectum may always be preferable [33], [41]. Although patients are currently given dietary advice and asked to clear their bowels before treatment, further precautions to minimise rectal gas, such as enemas, could be considered.

We have considered a "worst case" scenario, where the gas is circular, touches the rectal wall i.e. there was no solid matter between the gas and the rectal wall, and lies in the path of a single treatment beam. The dose effects due to ERE are reduced by around two thirds when in the path of multiple overlapping beams, compared to a single beam [14]. However, to gain a greater understanding of the absolute dose effects and increased risk of toxicity, this kind of assessment should be done using clinically realistic MRIgRT plans.

\section{Conclusion}

Rectal gas in bladder, prostate and cervical cancer patients is likely to remain stable for the duration of a 20-25 minute MRIgRT fraction. If not accounted for in the daily re-planning, the intrafractional stability of gas in abdominal cancer patients treated using MRIgRT is likely to cause relevant over-dosage due to ERE. However, the over-dosage can be mitigated if the gas is accounted for at the daily re-planning stage. This should be kept in mind when large volumes of rectal gas are seen in daily imaging at the beginning of treatment. Further investigation into factors that influence the volume and stability of rectal gas in different patient groups should be done in order to assess the likelihood of over-dosage or need for plan adaption, on a more personalised basis.

\section{References}

[1] A. Cree et al., "The Potential Value of MRI in External-Beam Radiotherapy for Cervical Cancer," Clin. Oncol., vol. 30, no. 11, pp. 737-750, Nov. 2018.

[2] L. Boldrini, D. Cusumano, F. Cellini, L. Azario, G. C. Mattiucci, and V. Valentini, "Online adaptive magnetic resonance guided radiotherapy for pancreatic cancer: state of the art, pearls and pitfalls.," Radiat. Oncol., vol. 14, no. 1, p. 71, Apr. 2019.

[3] M. van Vulpen, C. A. T. van den Berg, M. R. Moman, and U. A. van der Heide, "Difficulties and potential of correlating local recurrences in prostate cancer with the delivered local dose," Radiother. Oncol., vol. 93, no. 2, pp. 180-184, 2009. 
[4] S. Corradini et al., "MR-guidance in clinical reality: current treatment challenges and future perspectives," Radiat. Oncol., vol. 14, 2019.

[5] I. M. White et al., "Realizing the potential of magnetic resonance image guided radiotherapy in gynaecological and rectal cancer," Br. J. Radiol., p. 20180670, May 2019.

[6] A. Hunt, V. N. Hansen, U. Oelfke, S. Nill, and S. Hafeez, "Adaptive Radiotherapy Enabled by MRI Guidance," Clin. Oncol., vol. 30, no. 11, pp. 711-719, Nov. 2018.

[7] S. Acharya et al., "Online Magnetic Resonance Image Guided Adaptive Radiation Therapy: First Clinical Applications," Int. J. Radiat. Oncol. Biol. Phys., vol. 94, no. 2, pp. 394-403, 2016.

[8] R. de Crevoisier et al., "Changes in the Pelvic Anatomy After an IMRT Treatment Fraction of Prostate Cancer," Int. J. Radiat. Oncol. Biol. Phys., vol. 68, no. 5, pp. 1529-1536, Aug. 2007.

[9] A. Simon et al., "Is Dose Deformation-Invariance Hypothesis Verified in Prostate IGRT?," Int. J. Radiat. Oncol. Biol. Phys., vol. 97, no. 4, pp. 830-838, Mar. 2017.

[10] M. Sasaki et al., "Dose impact of rectal gas on prostatic IMRT and VMAT," Jpn. J. Radiol., vol. 33, no. 12, pp. 723-733, Dec. 2015.

[11] G. Eminowicz, J. Motlib, S. Khan, C. Perna, and M. McCormack, "Pelvic Organ Motion during Radiotherapy for Cervical Cancer: Understanding Patterns and Recommended Patient Preparation," Clin. Oncol., vol. 28, no. 9, pp. e85-e91, Sep. 2016.

[12] R. Jadon et al., "A Systematic Review of Organ Motion and Image-guided Strategies in External Beam Radiotherapy for Cervical Cancer," Clin. Oncol., vol. 26, no. 4, pp. 185196, Apr. 2014.

[13] L. Fokdal, H. Honoré, M. Høyer, P. Meldgaard, K. Fode, and H. Von Der Maase, "Impact of changes in bladder and rectal filling volume on organ motion and dose distribution of the bladder in radiotherapy for urinary bladder cancer," Int. J. Radiat. Oncol. Biol. Phys., vol. 59, no. 2, pp. 436-444, Jun. 2004.

[14] J. Shortall et al., "Assessing localised dosimetric effects due to unplanned gas cavities during pelvic MR-guided Radiotherapy using Monte Carlo simulations," Med. Phys., p. mp.13857, Oct. 2019.

[15] X. Chen, P. Prior, G.-P. Chen, C. J. Schultz, and X. A. Li, "Technical Note: Dose effects of $1.5 \mathrm{~T}$ transverse magnetic field on tissue interfaces in MRI-guided radiotherapy," Med. 
Phys., vol. 43, no. 8, pp. 4797-4802, 2016.

[16] A. J. E. Raaijmakers, B. W. Raaymakers, and J. J. W. Lagendijk, "Integrating a MRI scanner with a $6 \mathrm{MV}$ radiotherapy accelerator: dose deposition in a transverse magnetic field," Phys. Med. Biol. B Phys. Med. Biol, vol. 49, 2004.

[17] G. H. Bol, J. J. W. Lagendijk, and B. W. Raaymakers, "Compensating for the impact of non-stationary spherical air cavities on IMRT dose delivery in transverse magnetic fields.," Phys. Med. Biol., vol. 60, no. 2, pp. 755-68, 2015.

[18] a J. E. Raaijmakers, B. W. Raaymakers, and J. J. W. Lagendijk, "Integrating a MRI scanner with a $6 \mathrm{MV}$ radiotherapy accelerator: dose increase at tissue-air interfaces in a lateral magnetic field due to returning electrons.," Phys. Med. Biol., vol. 50, no. 7, pp. 1363-76, 2005.

[19] S. C. Morgan et al., "Hypofractionated Radiation Therapy for Localized Prostate Cancer: Executive Summary of an ASTRO, ASCO, and AUA Evidence-Based Guideline," Pract. Radiat. Oncol., vol. 8, pp. 354-360, 2018.

[20] D. Winkel et al., "Adaptive radiotherapy: The Elekta Unity MR-linac concept," Clin. Transl. Radiat. Oncol., vol. 18, pp. 54-59, Sep. 2019.

[21] D. Winkel et al., "Evaluation of Online Plan Adaptation Strategies for the 1.5T MR-linac Based on 'First-In-Man' Treatments," Cureus, vol. 10, no. 4, p. e2431, Apr. 2018.

[22] S. H. Hsu, I. Zawisza, K. O'Grady, Q. Peng, and W. A. Tomé, "Towards abdominal MRIbased treatment planning using population-based Hounsfield units for bulk density assignment," Phys. Med. Biol., vol. 63, no. 15, Jul. 2018.

[23] S. J. Hoogcarspel, J. M. Van der Velden, J. J. W. W. Lagendijk, M. van Vulpen, and B. W. Raaymakers, "The feasibility of utilizing pseudo CT-data for online MRI based treatment plan adaptation for a stereotactic radiotherapy treatment of spinal bone metastases.," Phys. Med. Biol., vol. 59, no. 23, pp. 7383-7391, Dec. 2014.

[24] J. H. Jonsson, M. G. M. Karlsson, M. G. M. Karlsson, and T. Nyholm, "Treatment planning using MRI data: an analysis of the dose calculation accuracy for different treatment regions.," Radiat. Oncol., vol. 5, no. 1, p. 62, Jun. 2010.

[25] J. J. E. Kleijnen et al., "Evolution of motion uncertainty in rectal cancer: implications for adaptive radiotherapy," Phys. Med. Biol., vol. 61, no. 1, pp. 1-11, 2016.

[26] J. Nijkamp et al., "Repeat CT assessed CTV variation and PTV margins for short- and 
long-course pre-operative RT of rectal cancer," Radiother. Oncol., 2012.

[27] M. Van Herk, "Errors and Margins in Radiotherapy," Semin. Radiat. Oncol., vol. 14, no. 1, pp. 52-64, 2004.

[28] A. Mcwilliam, J. Kennedy, C. Hodgson, E. V. Osorio, C. Faivre-Finn, and M. Van Herk, "Radiation dose to heart base linked with poorer survival in lung cancer patients," Eur. J. Cancer, vol. 85, pp. 106-113, 2017.

[29] J. Shortall et al., "Characterizing local dose perturbations due to gas cavities in Magnetic Resonance guided Radiotherapy," Med. Phys., p. mp.14120, Mar. 2020.

[30] J. Nijkamp, R. de Jong, J. J. Sonke, C. van Vliet, and C. Marijnen, "Target volume shape variation during irradiation of rectal cancer patients in supine position: Comparison with prone position," Radiother. Oncol., vol. 93, no. 2, pp. 285-292, Nov. 2009.

[31] J. Nijkamp, R. de Jong, J. J. Sonke, P. Remeijer, C. van Vliet, and C. Marijnen, "Target volume shape variation during hypo-fractionated preoperative irradiation of rectal cancer patients," Radiother. Oncol., vol. 92, no. 2, pp. 202-209, Aug. 2009.

[32] C. Fiorino et al., "Rectal and bladder motion during conformal radiotherapy after radical prostatectomy," Radiother. Oncol., vol. 74, no. 2, pp. 187-195, 2005.

[33] M. S. Hoogeman, M. Van Herk, J. De Bois, and J. V. Lebesque, "Strategies to reduce the systematic error due to tumor and rectum motion in radiotherapy of prostate cancer," Radiother. Oncol., vol. 74, no. 2, pp. 177-185, 2005.

[34] M. S. Hoogeman, M. Van Herk, D. Yan, L. J. Boersma, P. C. M. Koper, and J. V. Lebesque, "A model to simulate day-to-day variations in rectum shape," Int. J. Radiat. Oncol. Biol. Phys., vol. 54, no. 2, pp. 615-625, Oct. 2002.

[35] R. W. Chuter, A. Pollitt, P. Whitehurst, R. I. MacKay, M. van Herk, and A. McWilliam, "Assessing MR-linac radiotherapy robustness for anatomical changes in head and neck cancer," Phys. Med. Biol., vol. 63, no. 12, p. 125020, Jun. 2018.

[36] E. H. Huang et al., "Late rectal toxicity: dose-volume effects of conformal radiotherapy for prostate cancer," Int. J. Radiat. Oncol., vol. 54, no. 5, pp. 1314-1321, Dec. 2002.

[37] G. Dipasquale, T. Zilli, C. Fiorino, M. Rouzaud, and R. Miralbell, "Salvage reirradiation for local failure of prostate cancer after curative radiation therapy: Association of rectal toxicity with dose distribution and normal-tissue complication probability models," Adv. Radiat. Oncol., vol. 3, no. 4, pp. 673-681, Oct. 2018. 
[38] Y. Shiraishi et al., "Dose Constraint for Minimizing Grade 2 Rectal Bleeding Following Brachytherapy Combined With External Beam Radiotherapy for Localized Prostate Cancer: Rectal Dose-Volume Histogram Analysis of 457 Patients," Int. J. Radiat. Oncol., vol. 81, no. 3, pp. e127-e133, Nov. 2011.

[39] V. Beckendorf et al., "70 Gy Versus 80 Gy in Localized Prostate Cancer: 5-Year Results of GETUG 06 Randomized Trial," Int. J. Radiat. Oncol., vol. 80, no. 4, pp. 1056-1063, Jul. 2011.

[40] J. Adamson and Q. Wu, "Inferences About Prostate Intrafraction Motion From Pre- and Posttreatment Volumetric Imaging," Int. J. Radiat. Oncol. Biol. Phys., vol. 75, no. 1, pp. 260-267, Sep. 2009.

[41] I. Ogino, H. Uemura, T. Inoue, Y. Kubota, K. Nomura, and N. Okamoto, "Reduction of Prostate Motion by Removal of Gas in Rectum During Radiotherapy," Int. J. Radiat. Oncol. Biol. Phys., vol. 72, no. 2, pp. 456-466, Oct. 2008.

\section{Figure Captions}

Table 1: Summary of the patient images used in this study

Table 2: Rectal length variation results. The columns present the lengths used for normalisation in our analysis, the lengths of the delineated rectums, inter- and intra-scan-session and interpatient variation in rectal length.

Figure 1: A) Sagittal MRI images taken at seven time-points during one scan-session for one patient. The rectum delineations are outlined in red and the gas is overlaid in green. The pink lines enclose the slices where rectal delineation is common across all time-points, and the arrow in time-point 1 shows the direction of normalisation (0: most cranial, 1: most caudal). B) The axial views corresponding to the slice marked by the orange dotted line in panel $A$. The changes in intra-fractional gas (green) are apparent. C) The area of rectal gas $\left(\mathrm{cm}^{2}\right)$ in each slice of the normalised rectum for the same patient. Each line represents the gas distribution at different timepoints. The slice shown in panel $B$ is indicated by the dotted line.

Figure 2: Histograms showing the distribution of the volume of rectal gas in the planning CT scans of 70 cervical (panel A) 124 bladder (panel B), and 2,180 prostate (panel C) cancer patients. 
Figure 3: The area of rectal gas $\left(\mathrm{cm}^{2}\right)$ in each slice of the delineated rectum for cervical cancer patient 1. Each plot represents a different scan-session for the same patient, and each line represents the gas in a different time-point. The equivalent plots for all other patients are presented in Appendix A-C in supplementary material.

Figure 4: The local Group Mean $(M)$ and the systematic $(\Sigma)$ and random $(\sigma)$ inter- $(A-C)$ and intra$(D-F)$ fractional variation of rectal gas for each patient group. Intra-fractional random variations for each patient $\left(\sigma_{f}\right)$ averaged across all patients, are included in panels $D-F$.

Figure 5: The estimated dose perturbation (represented in a cylindrical coordinate system, similar

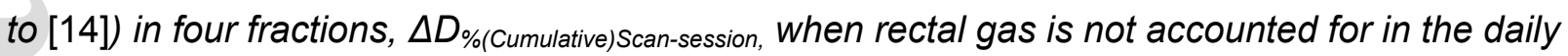
adaption during MRIgRT for cervical cancer patient 1 (in a single beam). The equivalent plots for all other patients are presented in Appendix A-C in supplementary material

Figure 6: The calculated cumulative dose perturbation in each scan-session, $\Delta D_{\%(\text { (Cumulative)Scan- }}$ session_adapt, when rectal gas is accounted for in the daily adaption during MRIgRT for cervical cancer patient 1. The equivalent plots for all other patients are presented in Appendix A-C in supplementary material.

Figure 7: Box and whisker plots showing the spread of the total dose perturbation for all scansessions on the internal surface of the rectal wall for each patient during a single beam treatment. The red boxes represent the dose perturbation when rectal gas is not accounted for in the daily adaption and the blue boxes represent the dose perturbation when the gas in the daily MRI (timepoint 1) has been accounted for in the daily plan. Panels A-C show the cumulative dose perturbation in each scan-session (overlaid on the same point on the $x$-axis for space purposes), and panels $D-F$ show the cumulative dose perturbation over all scan-sessions for each patient (four scan-sessions for cervix and bladder patients, 20 for prostate patients).

\section{Acknowledgements}

Funding: This work was supported by the European Association of National Metrology Institutes (EURAMET) as part of the European Metrology Programme for Innovation and Research (EMPIR) project [grant number R120635].

Conflicts of interest: The University of Manchester and The Christie are members of the Elekta MR-Linac consortium from which we have received financial and technical support under a research agreement with Elekta $A B$. However, Elekta had no part in the design or execution of the study.

This article is protected by copyright. All rights reserved 
Acknowledgements: Professor Marcel van Herk and Professor Ananya Choudhury were supported by NIHR Manchester Biomedical Research Centre. The Christie is supported by a Cancer Research UK centres Network Accelerator Award Grant (A21993) to the ART-NET consortium.

This article is protected by copyright. All rights reserved 
Table 1: Summary of the patient images used in this study

\begin{tabular}{|c|c|c|c|c|c|c|c|}
\hline Scan Type & Site & $\begin{array}{c}\text { Number of } \\
\text { patients }\end{array}$ & $\begin{array}{l}\text { Number of } \\
\text { scan-sessions }\end{array}$ & $\begin{array}{l}\text { Number of } \\
\text { time-points }\end{array}$ & Time scale & Scan preparations & Comments \\
\hline $\begin{array}{l}\text { MRI - } \\
\text { diagnostic } \\
\text { scanner }\end{array}$ & Cervix & 6 & 4 (weekly) & 7 & $\begin{array}{l}\text { Total } 20 \text { minutes } \\
\text { (2-3 minute intervals) }\end{array}$ & $\begin{array}{l}\text { Empty bladder, } 300 \mathrm{ml} \text { water } \\
\text { immediately before scan }\end{array}$ & 5 time-points for scan-session number 4 \\
\hline $\begin{array}{l}\text { MRI - } \\
\text { diagnostic } \\
\text { scanner }\end{array}$ & Bladder & 11 & 4 (weekly) & 3 & $\begin{array}{l}\text { Total } 25 \text { minutes } \\
\text { (10-14 minute intervals) }\end{array}$ & $\begin{array}{l}\text { Empty bladder, } 300 \text {-500 } \mathrm{ml} \text { water } \\
\text { immediately before scan } \\
\text { Hyoscine Butylbromide (Buscopan) } 30 \\
\text { mins prior to scan }\end{array}$ & $\begin{array}{l}\text { All patients were male } \\
2 \text { time-points for scan-session } 2 \text { for } \\
\text { patient } 1\end{array}$ \\
\hline $\begin{array}{l}\text { MRI - } \\
\text { Elekta Unity } \\
\text { system } \\
\text { (Elekta, } \\
\text { Stockholm, } \\
\text { Sweden) }\end{array}$ & Prostate & 5 & 20 (daily) & 3 & $\begin{array}{l}\text { Total } 30 \text { minutes } \\
\text { (10 minute intervals) }\end{array}$ & Empty bladder & $\begin{array}{l}\text { Only daily MRI available for patients 1, } 2 \\
\text { (two scan-session), } 3 \text { and } 5 \text { (one scan- } \\
\text { session) } \\
\text { Additional time-points for patient } 3 \text { (two } \\
\text { scan-sessions) and patient } 4 \text { (three scan- } \\
\text { sessions) (total time 60-75 minutes) }\end{array}$ \\
\hline
\end{tabular}

This article is protected by copyright. All rights reserved 


\begin{tabular}{|c|c|c|c|c|c|c|c|}
\hline \multirow{3}{*}{ Planning CT } & Cervix & 70 & 1 & - & - & - & Including 6 cervix patients who had MRI \\
\hline & Bladder & 124 & 1 & - & - & - & \\
\hline & Prostate & 2,180 & 1 & - & - & - & \\
\hline
\end{tabular}

This article is protected by copyright. All rights reserved 
Table 2: Rectal length variation results. The columns present the lengths used for

normalisation in our analysis, the lengths of the delineated rectums, inter- and intra-scansession and inter-patient variation in rectal length.

\begin{tabular}{|c|c|c|c|c|c|}
\hline & $\begin{array}{l}\text { Mean Rectum } \\
\text { length Analysed } \\
\text { (cm) }\end{array}$ & $\begin{array}{l}\text { Mean Rectum } \\
\text { Length Whole } \\
\text { (cm) }\end{array}$ & $\begin{array}{c}\text { Intra-Scan- } \\
\text { Session variation } \\
(\mathrm{cm})\end{array}$ & $\begin{array}{c}\text { Inter-Scan- } \\
\text { Session variation } \\
(\mathrm{cm})\end{array}$ & $\begin{array}{l}\text { Inter-patient } \\
\text { variation }(\mathrm{cm})\end{array}$ \\
\hline Cervix & 6.39 & 6.82 & 0.34 & 0.57 & 1.46 \\
\hline Bladder & 5.58 & 6.33 & 0.47 & 0.66 & 0.66 \\
\hline Prostate & 5.94 & 6.41 & 0.34 & 0.71 & 0.51 \\
\hline
\end{tabular}

This article is protected by copyright. All rights reserved 




B)

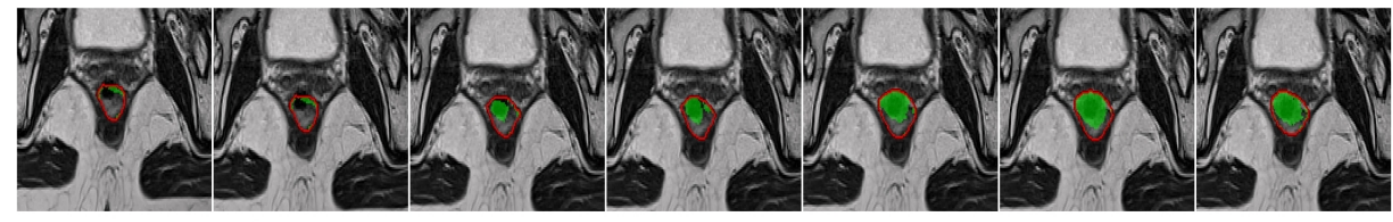

C)

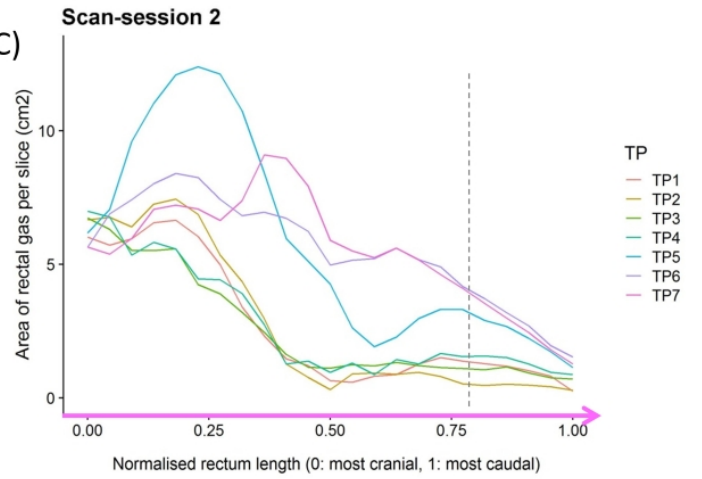

mp_14586_f1.jpg

This article is protected by copyright. All rights reserved 



mp_14586_f2.jpg 

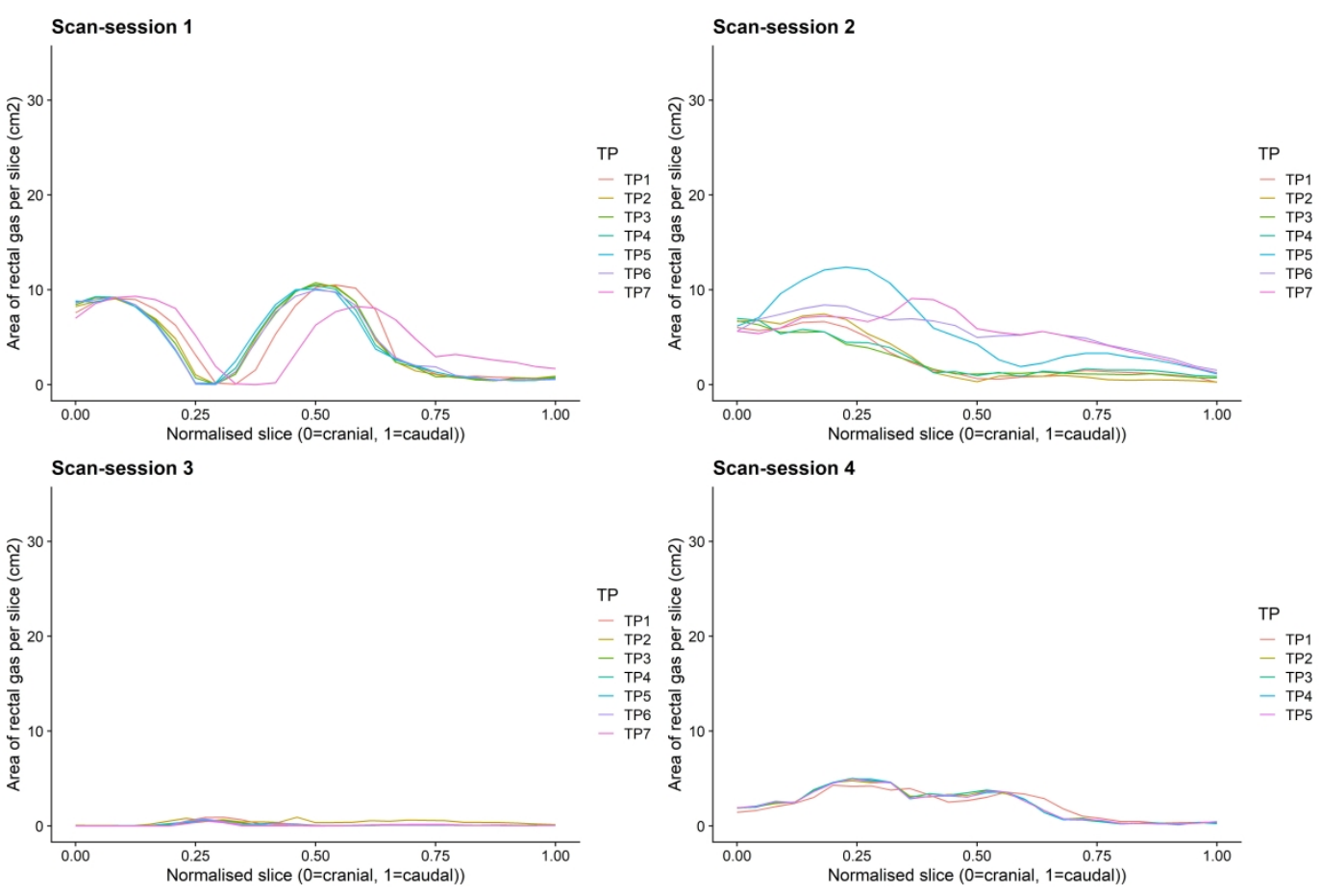

mp_14586_f3.jpg

This article is protected by copyright. All rights reserved 



mp_14586_f4.jpg 

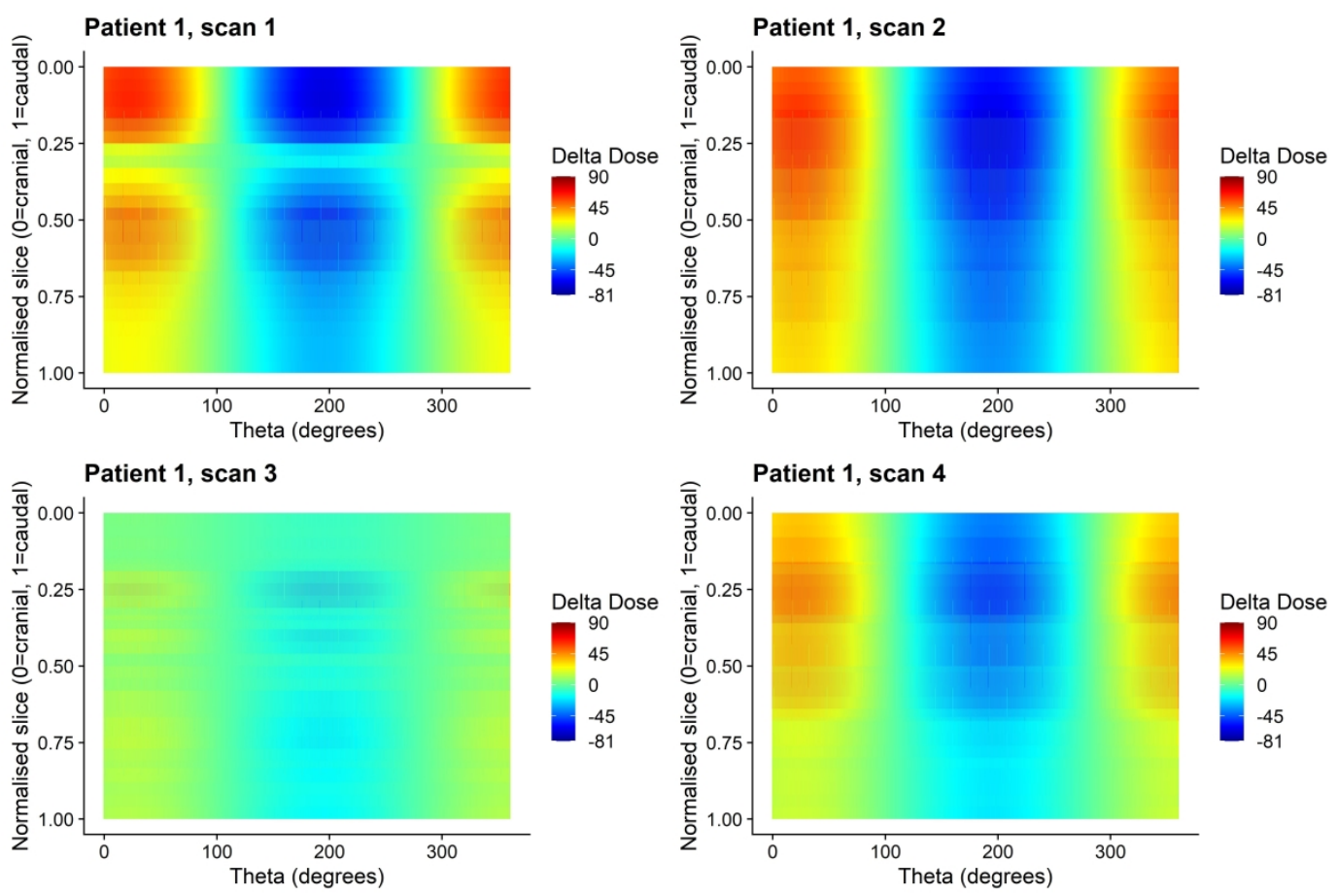

mp_14586_f5.jpg 



mp_14586_f6.jpg 


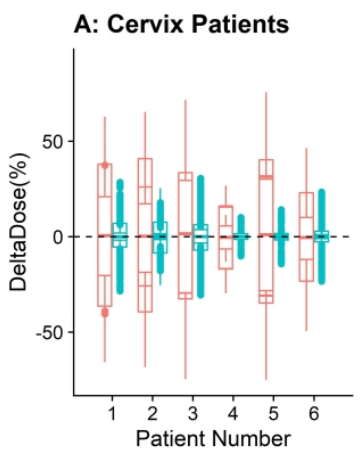

D: Cervix Patients

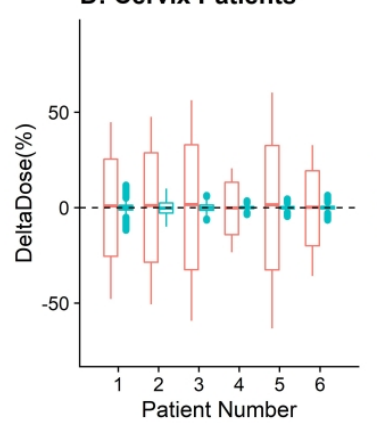

B: Bladder Patients

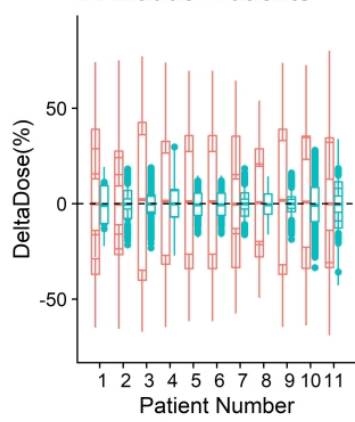

E: Bladder Patients

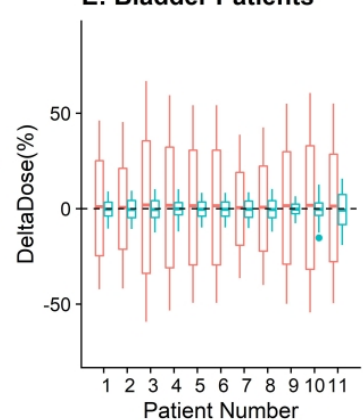

C: Prostate Patients


F: Prostate Patients
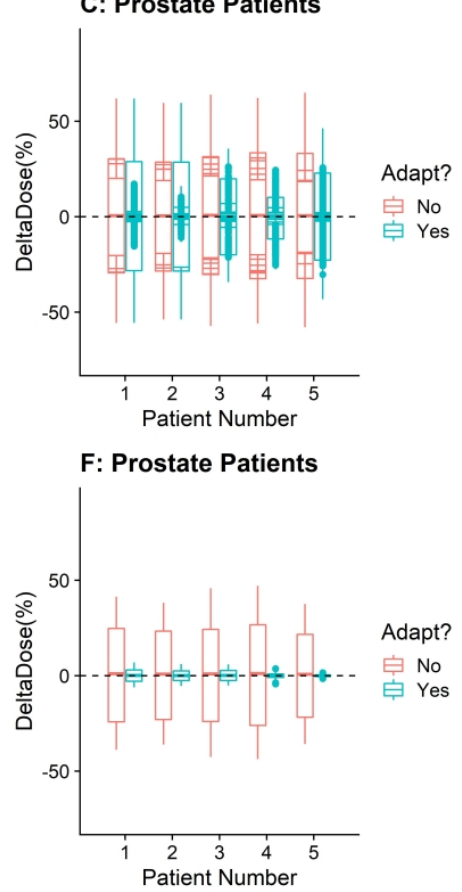

mp_14586_f7.jpg 